\title{
STRUCTURE CHANGES \\ IN Cz-Si SINGLE CRYSTALS IRRADIATED WITH FAST OXYGEN AND NEON IONS
}

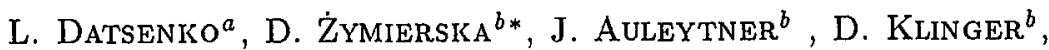 \\ V. MAChUlin ${ }^{a}$, V. KLAD'Ko ${ }^{a}$, V. Melnik ${ }^{a}$, I. ProKopenKo ${ }^{a}$, \\ T. CZOSNYKA ${ }^{c}$ AND J. CHOIŃSKI ${ }^{c}$
}

${ }^{a}$ Institute for Physics of Semiconductors, National Academy of Sciences of Ukraine

45, Nauki av., 252650 Kiev, Ukraine

${ }^{b}$ Institute of Physics, Polish Academy of Sciences

Al. Lotników 32/46, 02-668 Warsaw, Poland

${ }^{c}$ Heavy Ion Laboratory, Warsaw University, Pasteura 5a, 02-093 Warsaw, Poland

The research of the surface and the near-surface region of $\mathrm{Cz}-\mathrm{Si}$ wafers irradiated with fast oxygen and neon ions of energy $4 \mathrm{MeV} / \mathrm{u}$ and dose $10^{14}$ particles $/ \mathrm{cm}^{2}$ is presented. In our study several methods based on the Bragg case of X-ray diffraction using $\mathrm{Ag} K_{\alpha_{1}}$, as well as reflection high-energy electron diffraction and Nomarsky optical microscopy were used. It was shown that implantation with fast neon ions causes larger disturbances of silicon crystal structure than irradiation with oxygen ions.

PACS numbers: $61.80 . \mathrm{Jh}, 61.10 .-\mathrm{i}, 61.72 .-\mathrm{y}$

\section{Introduction}

The aim of the present paper is the research of peculiarities of defect structure of silicon crystals irradiated with swift ions of similar masses and energies but of distinguished character of interaction with $\mathrm{Si}$ atoms. For this purpose oxygen and neon ions were chosen. Due to its chemical activity the former is known to play an important role in forming $\mathrm{SiO}_{x}$ clusters which are the inner getters for undesirable impurities penetrating into bulk of the crystal by various technological processes, whereas the latter is neutral.

The present studies are undertaken in connection with the results of our previous paper [1], in which we reported the results of the X-ray measurements performed by means of a Philips high-resolution diffractometer using the characteristic $\mathrm{Cu} K_{\alpha_{1}}$ radiation $(\lambda=0.1541 \mathrm{~nm})$. From the recorded rocking curves the increase in the lattice parameter of silicon implanted with $4 \mathrm{MeV} / \mathrm{u}$ neon ions by

\footnotetext{
*Corresponding author, e-mail: zymier@ifpan.edu.pl
} 
$1 \times 10^{-3} \mathrm{~nm}$ was determined, whereas no change was noticed for silicon irradiated with oxygen ions with the same energy and dose, and from the grazing X-ray incidence reflectivity data, the increase in the surface roughness of implanted silicon crystals was detected.

In order to obtain an information from the deeper layers of the implanted silicon crystals we present in this paper the results of X-ray investigations carried out by using harder radiation, i.e. $\mathrm{Ag} K_{\alpha_{1}}$ radiation $(\lambda=0.0559 \mathrm{~nm})$.

\section{Experimental}

In the present research the complex methods based on the Bragg case of diffraction, i.e. a single crystal diffractometry for registration of spatial distribution (along the coordinate $x$ on a surface) of the diffracted beams intensity, and a double crystal diffractometry for measurement of the integral reflectivities $R_{\mathrm{i}}$ were used.

The two dislocation-free plates cut from a Czochralski-grown silicon $(\mathrm{Cz}-\mathrm{Si})$ crystal were implanted with $4 \mathrm{MeV} / \mathrm{u}$ oxygen and neon beams from the Warsaw Cyclotron [2-6], respectively. In both cases the beam current was equal to $50 \mathrm{enA}$ (electric nanoamper) and the total dose was about $10^{14}$ particles $/ \mathrm{cm}^{2}$. The implantation was performed by uniformly defocused beam at room temperature. The average ranges of oxygen ions and neon ions in silicon calculated using the programme TRIM 3 [7] are equal to $53.4 \mu \mathrm{m}$ and $46.2 \mu \mathrm{m}$, respectively. The third silicon sample was kept as a reference.

The half widths of the rocking curves as well as the integral reflectivities for the $220,440,660$, and 880 reflections were measured by using a double crystal $\mathrm{X}$-ray diffractometer in the dispersive geometry. The curves of spatial distribution of X-ray intensity were registered at the fixed Bragg position of the sample with narrow $(\approx 50 \mu \mathrm{m})$ slits situated near the moving detector. This method permits to separate from the recorded intensity the contribution of diffuse scattered intensity $I_{\mathrm{DS}}$ due to defects localized at various depths as well as elastic strains [8-10]. However, the inhomogeneous distribution of defects along the normal to the surface does not allow to determine some average structural characteristics of the layers situated at the absorption depth $t_{\mathrm{a}}=\mu^{-1}$, where $\mu$ is the photoelectric coefficient of absorption.

Furthermore, the reflection high-energy electron diffraction (RHEED) investigations were carried out by means of the EMR-102 diffractometer supplied with an electron gun working at $50 \mathrm{kV}$. The apparatus had a manipulator of samples which enabled to change the incidence angle of the electron beam within the large enough range of values and the azimuth angle in the interval $(0,2 \pi)$.

The surface studies of silicon samples were performed by using the interference-polarizing Nomarski microscope working in reflected light (magnification of the microscope objective was equal to $20 \mathrm{x}$ ).

\section{Results and discussion}

The dependence of measured integral reflectivities, $R_{\mathrm{i}}$, on the value of diffraction vector $\boldsymbol{H}$ (the reflection order) is presented in Fig. 1. For comparison the theoretical dependence (solid line) calculated for a perfect crystal using formulae 


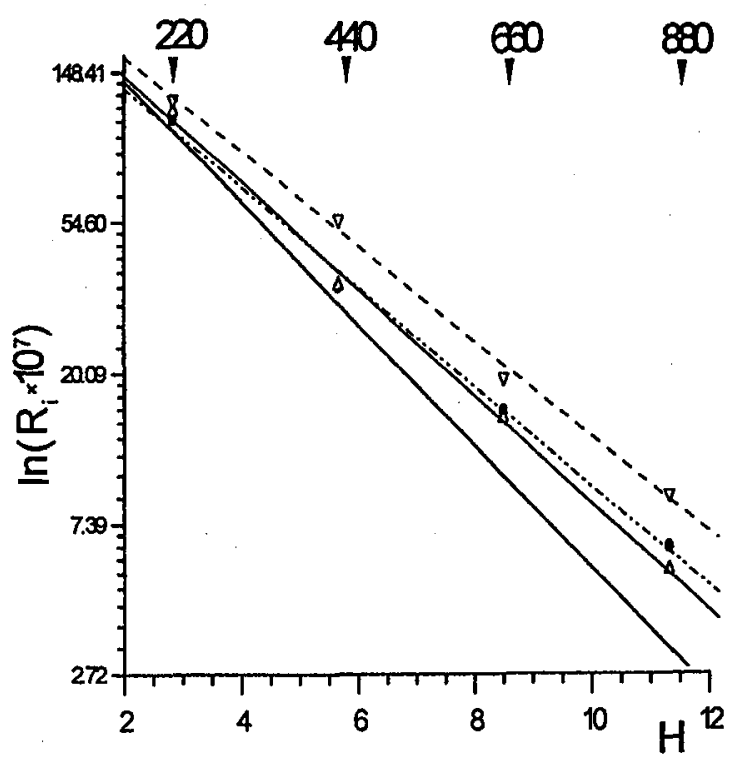

Fig. 1. Integral reflectivities $R_{\mathrm{i}}$ of Si crystals in the initial state ( $\bullet$ ) and after irradiation with $\mathrm{Ne}(\nabla)$ and $\mathrm{O}(\Delta)$ ions as a function of diffraction vector $\boldsymbol{H}$. The solid line presents the calculations using the X-ray dynamical theory for a perfect crystal. Upper numbers assign reflections.

of De Marco and Weiss [11] is shown. The extinction depth becomes larger with increasing the reflection order value, therefore information of the structural distortions localized at the larger depths can be obtained for these reflections. From Fig. 1 it follows that the low order reflection, 220, is weakly sensitive to defects, so the measured reflectivities of non-implanted sample as well as those for both irradiated crystals are close to each other and close to the theoretical one. The reflectivities of 660 and 880 reflections of the crystal irradiated with neon ions increase. The reflectivity of the 880 reflection deviates from the theoretical estimation even for the non-implanted sample owing to some grown-in microdefects of various nature.

The integrated reflectivity for the sample bombarded with oxygen ions did not increase but even became lower than that for the non-implanted sample. This is a curious fact because all of the known types of deformations of the structure cause an increment of $R_{\mathrm{i}}$. Therefore it is suggested that the average crystal lattice of the sample with defects becomes more perfect after irradiation with oxygen ions.

The rocking curves for investigated samples were also measured by using the double crystal diffractometer adjusted in dispersive geometry. It was stated that the half width of the rocking curve for 220 reflection decreases from $27 \operatorname{arcsec}$ to 20 arcsec. Therefore, one can suppose that some reconstruction of initial defect structure (relatively large $\mathrm{SiO}_{x}$ clusters) took place during processes of local heating of a lattice along the tracks of the slowing down oxygen ions which could 


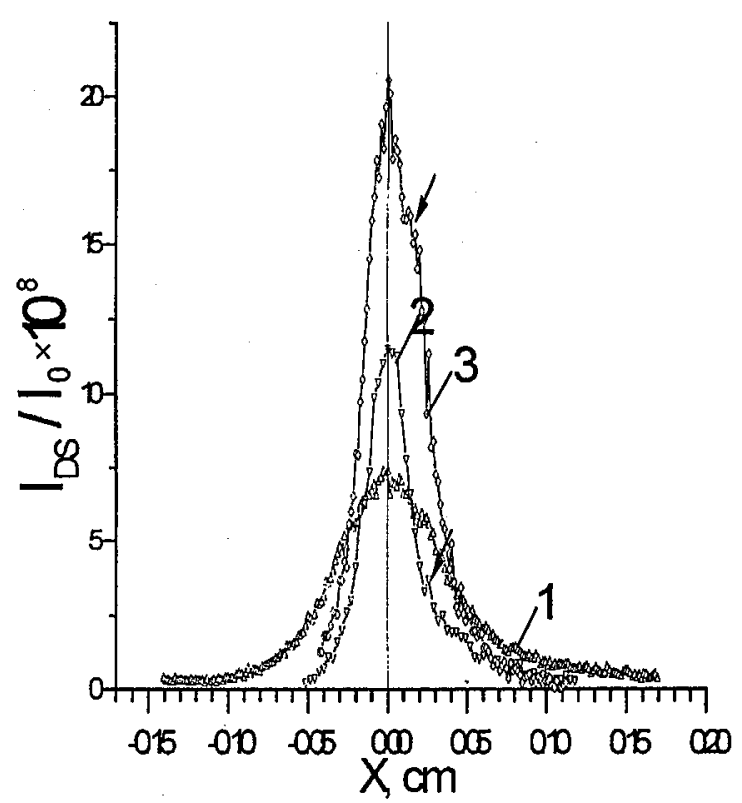

Fig. 2. Spatial intensity distributions of the Bragg diffracted beam (Ag $K_{\alpha_{1}}$ - radiation, 440 reflection, $x$ is the coordinate along the surface of the crystal from the incidence place of the initial beam with intensity $I_{0}$ ). The initial state is indicated by 1 , and the states after the implantation with the $\mathrm{O}$ and $\mathrm{Ne}$ ions by 2 and 3 , respectively. Arrows show the flash-up of intensity due to amorphous layers.

chemically interact with silicon atoms. During this structure reconstruction the energy of slowed down oxygen ions was spent for formation of small clusters which absorbed the excess of interstitial atoms.

For independent checking of the above hypothesis on the structure improving after irradiation of the sample with the oxygen ions, the curves of the spatial distribution of the Bragg diffracted beam intensities were considered. The analysis of shapes of these curves, which are shown in Fig. 2, performed basing on the interpretations given in papers [8-10] indicates for the larger variation of the shape of the curve after irradiation with neon ions. The diffraction effects (flash-up of intensity) from the amorphous layers are pointed out by the arrows on the curves for the both implanted samples. The enhancement of the intensities of scattered beams from the layers situated closely to the surface due to post-implantation defects is better noticeable for the sample irradiated with the oxygen ions than for the crystal bombarded with neon ions. The most curious fact is the narrowing of the intensity distribution for the sample irradiated with oxygen ions. Probably one can suppose that the thermal spikes [12] appearing at the ends of the oxygen ion tracks are those local regions where intensive diffusion processes occur resulting in the forming of the small nucleus of $\mathrm{SiO}_{x}$ clusters. An outflow of point defects from the regions surrounding the thermal spike happens more intensively in the case of the oxygen ions which interact chemically with silicon atoms, than in the case of neon ions. 

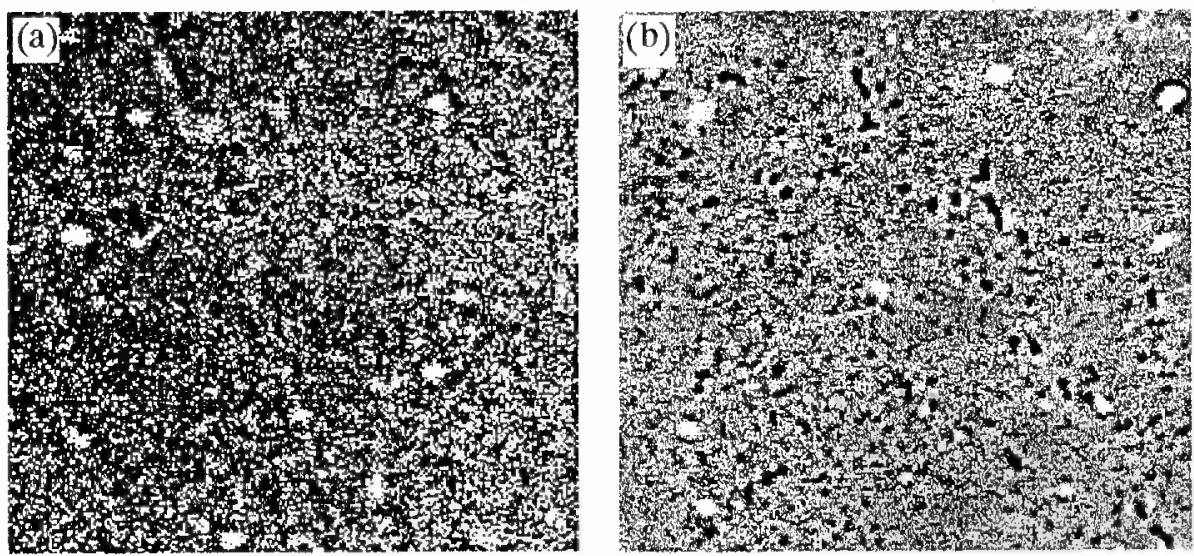

Fig. 3. The images from Nomarski optical microscope for silicon surfaces (magnification $400 \times$ ): (a) non-implanted surface, (b) surface of the sample irradiated with oxygen ions (visible black spots correspond to post-implantation defects).

Interference-polarizing optical microscopy investigations of the surface structure of all silicon samples have also been performed. The obtained images are shown in Fig. 3. The surface of the plate implanted with oxygen ions (see Fig. 3b) is characterised by the presence of surface post-implantation defects visible as black circle spots of $4 \mu \mathrm{m}$ diameter, whose density is high (about $6 \times 10^{3} \mathrm{~cm}^{-2}$ ).

The structural studies of non-implanted and implanted silicon samples carried out by RHEED technique at the incidence angle equal to $5^{\circ}$ refer to layers placed at the depths of a few nanometers, in order that the structural changes from the surface to these layers could be observed. The presence of Kikuchi lines confirms a nearly good structural quality of the near-surface region of investigated samples, even after irradiation.

\section{Conclusions}

The X-ray diffraction methods used in the present study have indicated the differences in our results concerning the implantation of the silicon crystals with swift oxygen and neon ions. For the sample implanted with neon ions a splash of the intensity caused by a distorted layer was visible. On the other hand, the improvement of crystal perfection in the case of crystal implanted with oxygen ions in comparison with the sample bombarded with neon ions was shown. It is suggested that the dissolution of grown-in microdefects ( $\mathrm{SiO}_{x}$ clusters) takes place during a chemical interaction of slowing down oxygen ions with silicon matrix.

The results of the X-ray methods were confirmed by the RHEED images indicating that the implantation with neon fast ions causes larger disturbance of silicon crystal structure than irradiation with oxygen ions. The differences between the surface structure of non-implanted and implanted with neon ions samples, and that of irradiated with oxygen ions are also stated by the images taken from the Nomarski optical microscope. The black circle spots visible in Fig. $3 b$ could be interpreted as $\mathrm{SiO}_{x}$ precipitates. 
The results of implantation with oxygen fast ions and those with neon fast ions differ due to the opposite character of chemical interaction of oxygen (active) from neon (neutral) with silicon matrix.

\section{Acknowledgment}

This study was partly supported by the Committee for Scientific Research (Poland) under the grant No. 2 P03B 10314.

\section{References}

[1] D. Żymierska, D. Klinger, J. Auleytner, T. Czosnyka, L. Datsenko, Nucl. Instrum. Methods Phys. Res. B 146, 350 (1998).

[2] Annual Report 1993, Heavy Ion Laboratory, Warsaw University, Warsaw 1993.

[3] Annual Report 1994, Heavy Ion Laboratory, Warsaw University, Warsaw 1994.

[4] Annual Report 1995, Heavy Ion Laboratory, Warsaw University, Warsaw 1995.

[5] Annual Report 1996, Heavy Ion Laboratory, Warsaw University, Warsaw 1996.

[6] Annual Report 1997, Heavy Ion Laboratory, Warsaw University, Warsaw 1997.

[7] G. Bardos, V.K. Fedyanin, J. Gavrilenko, Preprint of the Joint Institute for Nuclear Research, E 17-83-303, 1983.

[8] V.I. Khrupa, I.R. Entin, Metallofizika 13, 147 (1991) (in Russian).

[9] I.R. Entin, V.I . Khrupa, J. Appl. Crystallogr. 24, 403 (1991).

[10] J. Auleytner, V.I. Khrupa, L.I. Datsenko, S.M. Krasulya, M.J. Skorokhod, Acta Phys. Pol. A 89, 301 (1996).

[11] J.J. De Marco, R.J. Weiss, Acta Crystallogr. 19, 68 (1965).

[12] N.G. Van Bueren, Imperfections in crystals, North-Holland, Amsterdam 1960. 